\title{
Predicting the Grade of Prostate Cancer Based on a Biparametric MRI Radiomics Signature
}

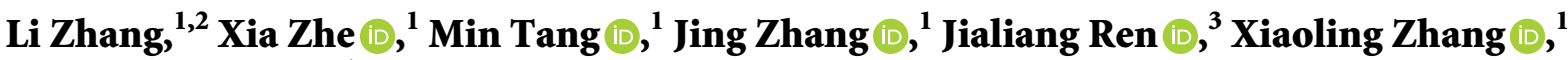 \\ and Longchao Li $\mathbb{D}^{1}$ \\ ${ }^{1}$ Department of MRI, Shaanxi Provincial People's Hospital, Xi'an, Shaanxi 710000, China \\ ${ }^{2}$ Department of Radiology, Xijing Hospital, Fourth Military Medical University, 17 Changle Road, Xi'an, Shaanxi 710032, China \\ ${ }^{3}$ GE Health Care, Beijing 100000, China
}

Correspondence should be addressed to Longchao Li; lxllc@126.com

Received 18 August 2021; Revised 8 October 2021; Accepted 3 December 2021; Published 23 December 2021

Academic Editor: J.M. Gorriz

Copyright (c) $2021 \mathrm{Li}$ Zhang et al. This is an open access article distributed under the Creative Commons Attribution License, which permits unrestricted use, distribution, and reproduction in any medium, provided the original work is properly cited.

Purpose. This study aimed to investigate the value of biparametric magnetic resonance imaging (bp-MRI)-based radiomics signatures for the preoperative prediction of prostate cancer $(\mathrm{PCa})$ grade compared with visual assessments by radiologists based on the Prostate Imaging Reporting and Data System Version 2.1 (PI-RADS V2.1) scores of multiparametric MRI (mp-MRI). Methods. This retrospective study included 142 consecutive patients with histologically confirmed PCa who were undergoing mpMRI before surgery. MRI images were scored and evaluated by two independent radiologists using PI-RADS V2.1. The radiomics workflow was divided into five steps: (a) image selection and segmentation, (b) feature extraction, (c) feature selection, (d) model establishment, and (e) model evaluation. Three machine learning algorithms (random forest tree (RF), logistic regression, and support vector machine (SVM)) were constructed to differentiate high-grade from low-grade PCa. Receiver operating characteristic (ROC) analysis was used to compare the machine learning-based analysis of bp-MRI radiomics models with PI-RADS V2.1. Results. In all, 8 stable radiomics features out of 804 extracted features based on T2-weighted imaging (T2WI) and ADC sequences were selected. Radiomics signatures successfully categorized high-grade and low-grade PCa cases $(P<0.05)$ in both the training and test datasets. The radiomics model-based RF method (area under the curve, AUC: 0.982; 0.918), logistic regression (AUC: $0.886 ; 0.886$ ), and SVM (AUC: $0.943 ; 0.913$ ) in both the training and test cohorts had better diagnostic performance than PI-RADS V2.1 (AUC: 0.767; 0.813) when predicting PCa grade. Conclusions. The results of this clinical study indicate that machine learning-based analysis of bp-MRI radiomic models may be helpful for distinguishing high-grade and low-grade PCa that outperformed the PI-RADS V2.1 scores based on mp-MRI. The machine learning algorithm RF model was slightly better.

\section{Introduction}

Prostate cancer $(\mathrm{PCa})$ is the most common malignancy in men [1]. Based on the Gleason score (GS), PCa is categorized as low-grade or high-grade, for which the treatment strategies differ greatly [2]. For instance, patients with high-grade $\mathrm{PCa}$ need radical prostatectomy or radiation therapy, whereas patients with low-grade PCa might be candidates for active surveillance [3-6]. Therefore, a preoperatively accurate prediction of the grade of $\mathrm{PCa}$ is critical for treatment decision-making.
Currently, biopsy is the reference standard for preoperatively identifying the grade of $\mathrm{PCa}$ [7]. However, this procedure has been shown to be susceptible to overdetection of low-grade and underdiagnosis of high-grade PCa [8]. Therefore, developing a noninvasive and accurate approach for the preoperative prediction of grade is desirable.

Multiparametric (Mp)-MRI has been recognized as a complementary tool for the detection and assessment of PCa. Radiologists use the Prostate Imaging Reporting and Data System Version 2.1 (PI-RADS V2.1) to detect clinically significant PCa [9]. Nevertheless, mp-MRI interpretation is 
challenging and prone to inter- and intrareader variability among expert radiologists [8].

According to the literature, machine learning-based $\mathrm{mp}$ MRI radiomics provide an objective tool and have been shown to be helpful in assessing the grade of PCa [10-12]. In contrast, dynamic contrast enhancement (DCE) is a timeconsuming process with additional costs of contrast agents [13-15]. Additionally, a meta-analysis reported that DCEMRI cannot be used to predict GS in PCa [16].

To our knowledge, few studies have predicted grades using biparameter (bp)-MRI (noncontrast agent) with radiomics [17]. However, machine learning algorithms are relatively simple, and the results of radiomics have not been compared with the traditional PI-RADSV2.1 approach. Thus, the aim of this study is twofold. First, we used three machine learning methods of radiomics based on bp-MRI, including logistic regression, random forests (RF), and support vector machines (SVM), to preoperatively predict PCa grade. A second purpose was to compare these classifying capabilities to the visual assessments of the radiologists based on mp-MRI of the PI-RADS V2.1 protocol.

\section{Materials and Methods}

The local institutional review board approved this retrospective cohort study and waived the requirement for written informed consent.

2.1. Patients. Between January 2017 and November 2020, a total of 166 consecutive patients who underwent prostate mp-MRI examinations with histologically confirmed PCa were included in this study. The exclusion criteria were as follows: (a) patients who received prior treatment including hormonal irradiation before the MRI scans $(n=21)$; (b) poor quality of the MRI images due to severe susceptibility artifacts or respiratory motion artifacts $(n=2)$; and (c) incomplete clinical data $(n=1)$.

Ultimately, 142 patients with PCa were enrolled in this study. For patients with multiple tumor sites, the site with the largest burden (namely, the largest size or the highest GS) was reported in the document. Therefore, in this study, only one tumor site from each patient was used for analysis [17].

According to recent radiomics studies [18], these enrolled patients were randomly divided into a training cohort $(n=98)$ and a test cohort $(n=44)$ using computer-generated random numbers at a $7: 3$ ratio. Flow diagram of patient recruitment is shown in Figure 1.

2.2. MRI Protocol and Feature Extraction. All images were acquired using a 3.0 TMR scanner (Achieva TX, Philips Healthcare, and the Netherlands) with a 16-channel body phased array coil. A standard mp-MRI protocol included sagittal T2WI, axial T2WI, diffusion-weighted imaging (DWI) (b values of 0 and 1000, $2000 \mathrm{sec} / \mathrm{mm}^{2}$ ), and DCE. Apparent diffusion coefficient (ADC) maps were automatically reconstructed on a designated workstation. The

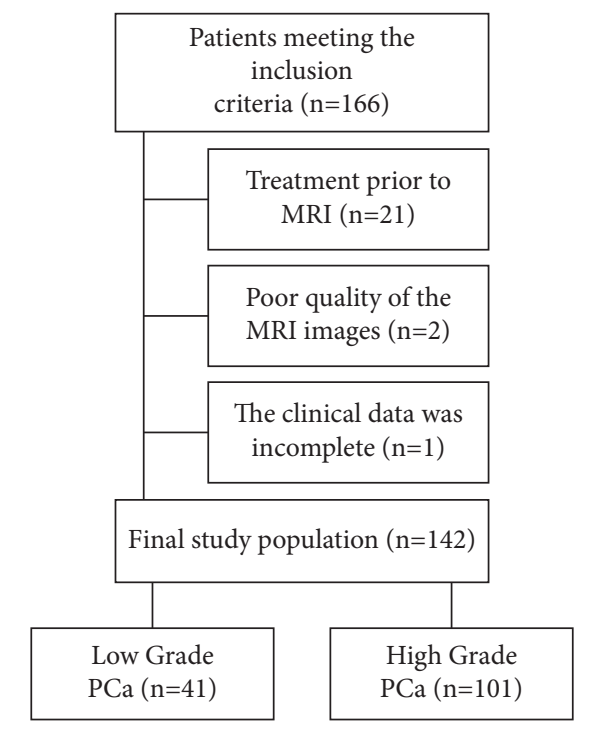

FIGURE 1: Flow diagram of patient recruitment.

detailed acquisition parameters of the MRI sequences are shown in Table 1.

Both the ADC and T2WI images were retrieved from a picture archiving and communication system (PACS, Huahai). The MRI images were loaded into ITK-SNAP software (version 3.4.0; https://www.itksnap.org) semiautomatically, and a three-dimensional volume of interest (VOI) that covered the whole tumor was delineated on both the axial ADC and T2WI images on each slice segmented by one radiologist (radiologist C, Z. L., with 10 years of experience in prostate MRI). The procedure of VOI is shown in Figure 2.

Texture extraction was performed using artificial intelligence kit (A.K., v. 3.2.1, GE Healthcare) software. In total, 804 imaging features were extracted from each VOI, including first-order statistics, histogram features, secondorder textures, and form factor (shape) parameters.

Then, interclass correlation coefficients (ICCs) were used to assess the interobserver reproducibility of radiomic feature extraction. Approximately 30 randomly chosen images were obtained by a senior radiologist (radiologist D, Z. X. L., with 20 years of experience in prostate MRI) for VOI segmentation.

2.3. Feature Selection, Radiomics Signature Construction, and Model Training. Not all the extracted features were useful for differential diagnosis. The abovementioned features were selected through least absolute shrinkage selection operator (LASSO) regression analysis with a 10 -fold cross-validation and Spearman correlation (threshold 0.9) coefficient for dimensionality reduction and feature selection to optimize the size of a feature set and to keep features independent.

Finally, 8 features were extracted from the T2WI and ADC images. The process of feature selection using the LASSO algorithm is shown in Figure 3.

The goal of the machine learning routine was to construct a predictive model to discriminate between the two 
TABLE 1: The detailed acquisition parameters of the MRI sequences.

\begin{tabular}{lccc}
\hline Parameters & Axial T2WI & DWI $(b=50,1000$, and 2000$)\left(\mathrm{sec} / \mathrm{mm}^{2}\right)$ & DCE \\
\hline Field of view (mm) & $200 \times 200$ & $220 \times 220$ & $260 \times 260$ \\
Acquisition matrix & $372 \times 363$ & $88 \times 82$ & $216 \times 217$ \\
Repetition time (ms) & 3020 & 3960 & 3.3 \\
Echo time (ms) & 100 & 86 & 1.59 \\
Flip angle (degree) & 90 & 90 & 10 \\
Section thickness (mm), no gaps & 3 & 3 & 2 \\
Image reconstruction matrix (pixel) & $339 \times 339$ & $160 \times 160$ & $288 \times 288$ \\
Reconstruction voxel imaging resolution (mm/pixel) & $0.34 \times 0.34 \times 4$ & $1.63 \times 1.63 \times 4$ & $0.9 \times 0.9 \times 2$ \\
\hline
\end{tabular}

Mp-MRI, multiparametric MRI; T2WI, T2-weighting imaging; DWI, diffusion weighted imaging; DCE, dynamic contrast-enhanced.
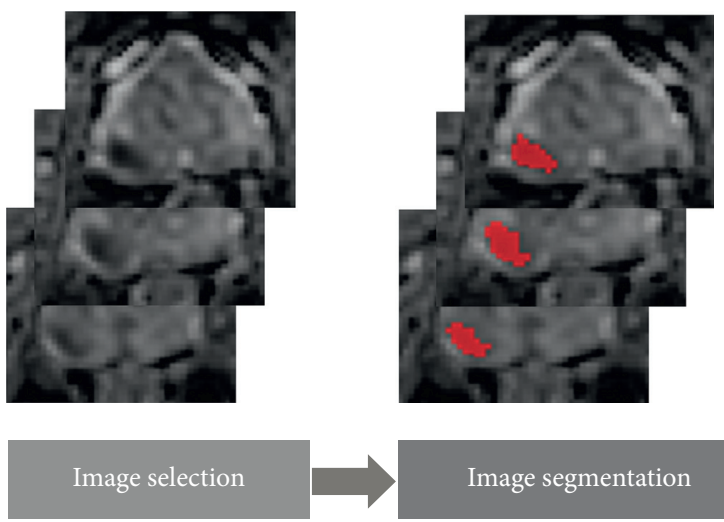

\section{Image segmentation}

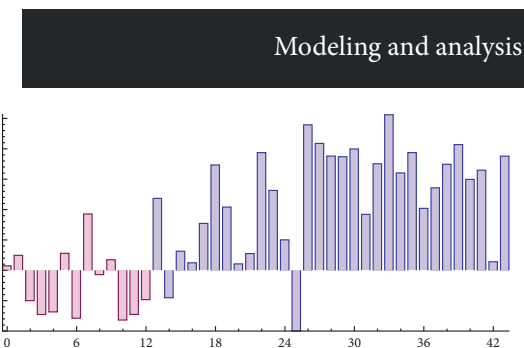

Radiomics signature
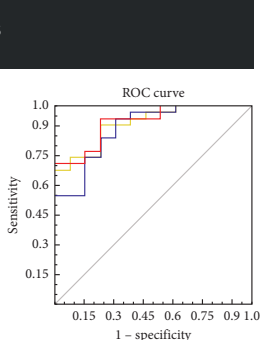

- ${ }_{\mathrm{SVM}}^{\mathrm{RF}}$

二 Logistic Regression
ROC curve
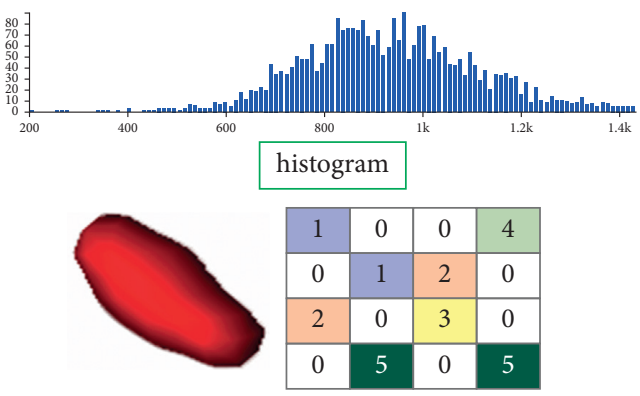

shape

texture
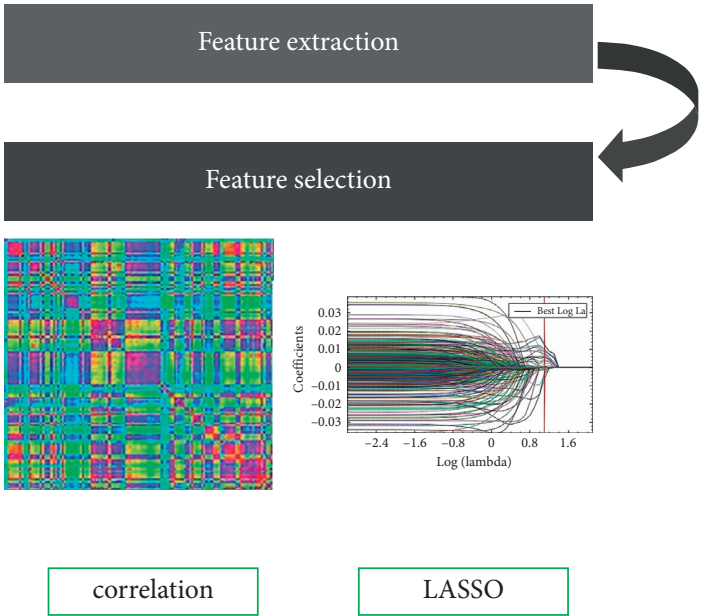

FIgURe 2: Radiomics workflow and study flowchart.

classes: low-grade and high-grade PCa. Three algorithms (RF, logistic regression, and SVM) were proposed as classifiers [19-21]. They can be used to find worthwhile features and remove relatively insignificant features to achieve higher classification performance [22-26]. The model assessed in the training dataset was applied to the test cohort. The radiomics workflow is presented in Figure 2, and the process is outlined in Figure 4.

2.4. PI-RADS V2.1 Evaluation. All images were networked to a communication workstation (Huahai Medical Imaging PACS, Xi'an, China) and Philips workspaces. According to the PI-RADS V2.1 guideline, two independent radiologists with different levels of experience (radiologist A, Z. J., with 3 years of experience, and radiologist B, T. M., with 10 years of experience in prostate MRI diagnosis), who were blinded to the initial mp-MRI imaging reports, clinical data, and histopathology, scored the examinations. In addition, the two radiologists did not previously participate in the process of VOI delineation. The PI-RADS V 2.1 score was independently recorded by radiologists using a score of $1-5$ for T2WI, a score of 1-5 for DWI, a “+” or “-” for DCE, and an overall PI-RADS assessment category. When multiple doubtful lesions appeared in the same patient, only the most suspicious lesion with the maximum volume was scored and recorded [27]. Lesions graded as having a PI-RADS $\geq 4$ were considered positive for high-grade $\mathrm{PCa}$, and lesions with a score $\leq 3$ were considered negative for low-grade PCa. The end performance for both radiologist and the radiomics 


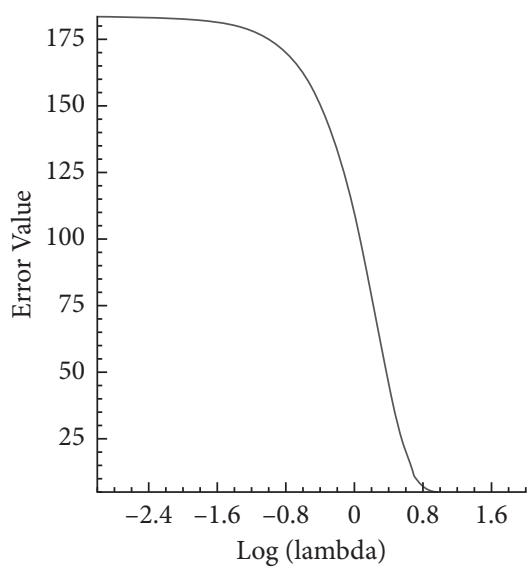

(a)

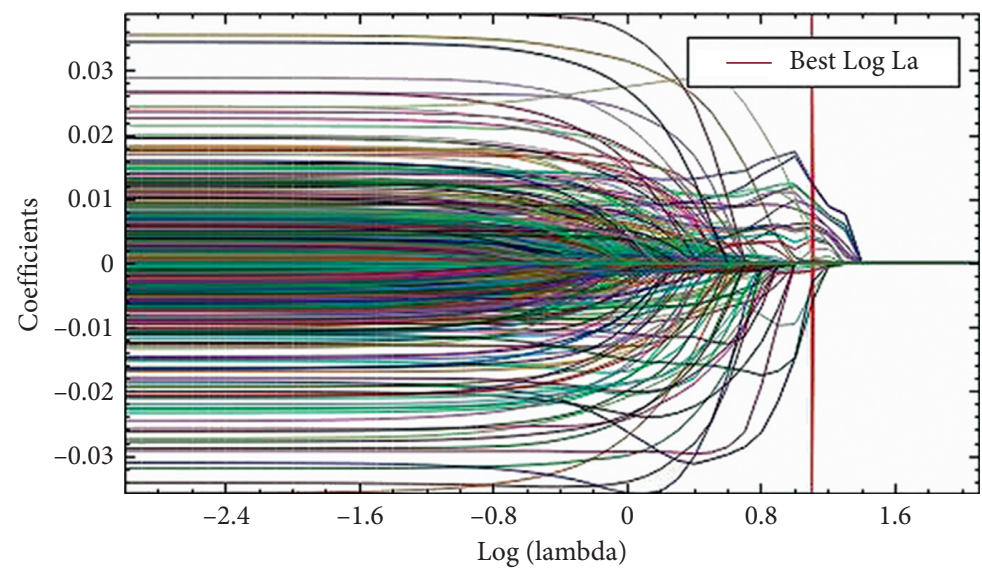

(b)

FIgURE 3: The process of feature selection using the LASSO algorithm. (a) The optimal tuning parameter (lambda) in the LASSO model was selected using 10-fold cross-validation and the 1 standard error rule. (b) LASSO coefficient profiles of the 26 features. The vertical line was drawn according to the 10-fold cross-validation in (a). LASSO, least absolute shrinkage selection operator.

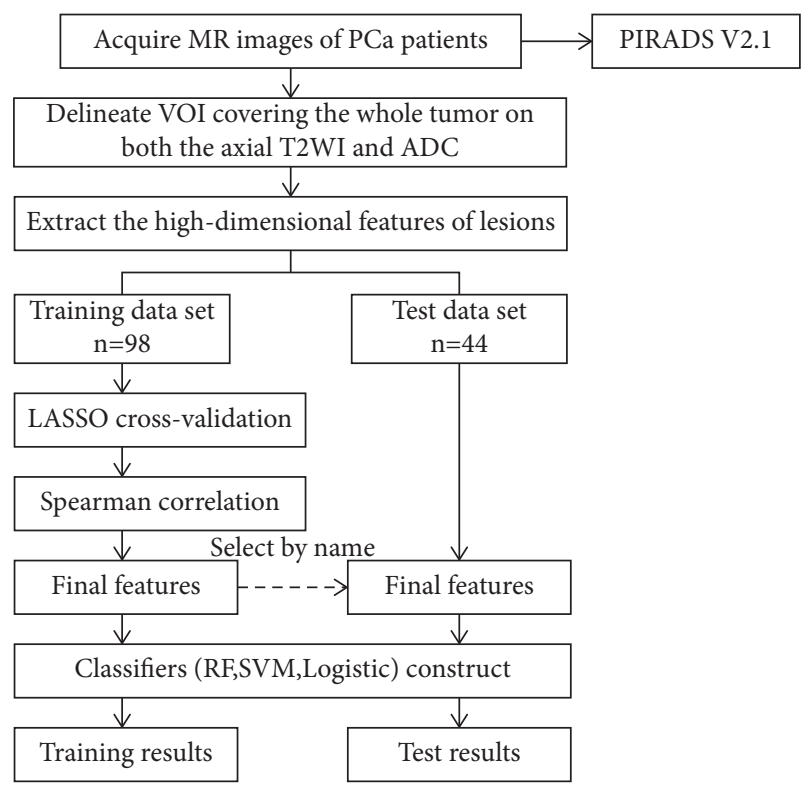

Figure 4: The process of the study.

model was computed based on patient-level classification. The detailed evaluation items of PI-RADS V2.1 scale (Supplement Tables 1-5) are uploaded in the supplementary materials.

2.5. Statistical Analysis. A kappa test was used to assess the inter-reader agreement of the PI-RADS V2.1 scores obtained by the two radiologists. Kappa values $<0.20$ indicated poor agreement, 0.21-0.40 indicated fair agreement, 0.41-0.60 indicated moderate agreement, $0.61-0.80$ indicated good agreement, and $\geq 0.81$ indicated excellent agreement [28]. The ICC was used to evaluate the interobserver reproducibility of the extracted radiomic features. An ICC score greater than 0.75 indicates good agreement of the feature extraction. The training and test cohorts were used to verify the diagnostic performance of the prediction models with RF, logistic regression, and SVM. The predictive ability of the radiomics signature models based on bp-MRI vs. mpMRI of the PI-RADS V2.1 score in identifying low-grade and high-grade PCa was analyzed based on receiver operating characteristic (ROC) curves. The performance of radiomics models was compared by the DeLong test [29]. The areas under the curve (AUC), sensitivity, specificity, positive likelihood ratio (LR+), and negative likelihood ratio (LR-) were derived in both the training and test cohorts. Highgrade tumors were considered the positive class.

$P<0.05$ indicates statistical significance. The statistical analysis was performed with the Statistical Package for the Social Sciences (SPSS, https://www.ibm.com/products/spssstatistics), MedCalc software, and A.K. software (mentioned above).

\section{Results}

3.1. Clinicopathologic Characteristics of Patients. The baseline characteristics of selected patients whose data were classified into the training and test cohort are summarized in Table 2. There were no significant differences in terms of age or PSA density (PSAD) between the two groups (all $P>0.05)$. Significance differences between high-grade and low-grade PCa were found in PSA level in the training and test cohorts (all $P<0.05$ ). The difference in location was statistically significant in the training cohort $(P<0.05)$.

3.2. Radiomics Signature Construction. The reliability of the extracted radiomic features in terms of ICC for all features of ADC and T2WI images was quantified, with mean ICC values of 0.919 (95\% CI: $0.836-0.960)$ and 0.963 (95\% CI: 0.925-0.982), respectively.

A total of 804 quantitative features were extracted from the VOI of each of the MRI series and their corresponding filtered results. 
TABLE 2: The baseline characteristics of selected patients in the training and test cohorts.

\begin{tabular}{|c|c|c|c|c|c|c|}
\hline \multirow[b]{2}{*}{ Characteristics } & \multicolumn{3}{|c|}{ Training cohort $(n=98)$} & \multicolumn{3}{|c|}{ Test cohort $(n=44)$} \\
\hline & $\begin{array}{l}\text { High-grade PCa } \\
\quad(n=70)\end{array}$ & $\begin{array}{l}\text { Low-grade PCa } \\
\quad(n=28)\end{array}$ & $P$ value & $\begin{array}{c}\text { High-grade PCa } \\
\quad(n=31)\end{array}$ & $\begin{array}{l}\text { Low-grade PCa } \\
\quad(n=13)\end{array}$ & $P$ value \\
\hline Mean age $(y)$ & $73.19 \pm 8.56$ & $72.11 \pm 7.58$ & 0.562 & $74.77 \pm 8.245$ & $72.46 \pm 8.353$ & 0.403 \\
\hline $\begin{array}{l}\text { Median PSA } \\
(\mathrm{ng} / \mathrm{ml})\end{array}$ & 16.47 (IQR:8.21-61.78) & 8.28 (IQR:6.36-14.03) & $<0.05$ & $\begin{array}{c}15.3481(\mathrm{IQR} \\
8.28-62.1)\end{array}$ & 8.81 (IQR:6.27-14.41) & $<0.05$ \\
\hline $\begin{array}{l}\text { Median PSAD } \\
(\mathrm{ng} / \mathrm{mL} / \mathrm{g})\end{array}$ & 0.15 (IQR:0.08-0.17) & 0.15 (IQR:0.07-0.14) & 0.76 & 0.20 (IQR:0.08-0.28) & 0.18 (IQR:0.11-0.21) & 0.7 \\
\hline \multicolumn{7}{|l|}{ Location } \\
\hline PZ & 25 & 13 & & 11 & 8 & \\
\hline $\mathrm{TZ}$ & 9 & 11 & $<0.05$ & 3 & 4 & 0.11 \\
\hline $\mathrm{PZ}$ and $\mathrm{TZ}$ & 36 & 4 & & 17 & 1 & \\
\hline
\end{tabular}

Date are mean $\pm \mathrm{SD}$. IQR, interquartile range; TZ, transition zone; PZ, peripheral zone; PSAD, PSA density.

In the current study, 8 features were obtained from bpMRI (constructed from 8 optimal feature sets selected from 804 features combined by T2WI and ADC sequences). The process of feature selection using the selection step is shown in Table 3.

A machine learning-based analysis of the bp-MRI radiomics signatures was established based on T2WI combined with ADC images for (a) RF; (b) logistic regression; and (c) SVM model.

\subsection{Predictive Ability of the Radiomics Signatures (Models).} The results show that the differential diagnostic model established using the RF method (AUC: $0.982 ; 0.918$ ) performed better than logistic regression analysis (AUC: 0.886 ; 0.886) and SVM (AUC: $0.943 ; 0.913$ ) in both the training and test cohorts. The DeLong test showed that the RF method presented a better AUC than logistic regression analysis in training cohorts $(P<0.05)$. The ROC curves of the radiomic signatures for discriminating performance in both the training and test cohorts are shown in Figure 5. The predictive ability (AUC, sensitivity, specificity, LR+, and LR-) of the radiomics model is shown in Table 4 for the training and test groups (see Supplementary Materials for the original radiomics data).

The kappa coefficient of the two radiologists was 0.7 , which indicated good agreement. Based on a PI-RADS V2.1 with a score of 5 as the cutoff value, the AUCs of the two radiologists' diagnosis performances were 0.767 and 0.813 , respectively, which were lower than those of the radiomics feature classifiers in classifying high-grade and low-grade PCa (Figure 5 and Table 4). Table 5 lists the results of the evaluations of all images by the two radiologists. The scores for each case are based on the PI-RADS V2.1 scale of radiologists $\mathrm{A}$ and $\mathrm{B}$, which are uploaded in the supplementary materials (Supplement Table 6).

\section{Discussion}

Preoperative prediction of PCa grade is important for clinical decision-making. Pathological biopsy is a popular tool for the estimation of PCa grade invasiveness, but it has various complications that limit its clinical use.
In this study, we described a radiomics signature using noncontrast-enhanced MRI images, which is a new noninvasive method with good diagnostic performance. Our results showed that the machine learning-based analysis of bp-MRI-based radiomics models for distinguishing lowgrade from high-grade PCa provided higher accuracy and sensitivity than visual assessments by radiologists based on the PI-RADS V2.1 mp-MRI score. This indicates that T2WI or ADC images could reflect the heterogeneity of tumors and that radiomic features can be used to distinguish small signal differences in prostate tumors. The present study also found that selecting the appropriate machine learning algorithms may help improve model stability and prediction performance. The results of our study show that the radiomicsbased RF machine-learning models, which incorporated 8 selected features, have better discrimination performance than other models (SVM and logistic regression) for differentiating low-grade from high-grade PCa (AUC of the training cohort: 0.982; AUC of the test cohort: 0.918 ) in both the training and test cohorts.

Some studies suggest the advantages of logistic regression models in aggressive PCa $[30,31]$. Generally, logistic regression, RF, and SVM algorithms are all suitable for model construction with small sample sizes and binary variables [30-34]. However, for bp-MRI (ADC combined with T2WI), the RF algorithm is more recommended for use with our small sample result.

We also assessed the predictive values of mp-MRI based on PI-RADS V2.1. In our study, the predicted probabilities of the radiomics features outperformed subjective evaluation by the human group.

Previous studies have evaluated the performance of MRI radiomic-based models for predicting aggressive PCa. A study by Parra et al. explored DCE and ADC-feature radiomics models for predicting clinically significant $\mathrm{PCa}$ with an AUC of 0.82 [35]. Ma $S$ et al. reported that an mpMRI radiomics signature that outperformed the radiologists' visual assessments in predicting extracapsular extension was developed and validated [36]. Niu et al. reported that a texture analysis-based model that includes bp-MRI can be used for identifying high-grade PCa and that specific parameters extracted from texture analysis may be additional tools for assessing tumor aggressiveness [37]. Min et al. 
TABLE 3: The process of feature selection using the selection step.

Lasso: cross validation

"Min intensity"

"Histogram entropy"

"Correlation_AllDirection_offset1_SD"

"GLCMEntropy_AllDirection_offset1"

"GLCMEntropy_AllDirection_offset4"

"GLCMEntropy_AllDirection_offset7"

"GLCMEntropy_angle0_offset1"

"GLCMEntropy_angle0_offset4"

"GLCMEntropy_angle0_offset7"

"GLCMEntropy_angle135_offset1"

"GLCMEntropy_angle135_offset4"

"GLCMEntropy_angle135_offset7"

"GLCMEntropy_angle45_offset1"

"GLCMEntropy_angle45_offset4"

"GLCMEntropy_angle90_offset1"

"GLCMEntropy_angle90_offset4"

"GLCMEntropy_angle90_offset7"

"Hara entroy"

"Sum average"

"Sum entropy"

"ShortRunHighGreyLevelEmphasis_AllDirection_offset1"

"ShortRunHighGreyLevelEmphasis_angle0_offset1"

"ShortRunHighGreyLevelEmphasis_angle0_offset7"

"ShortRunHighGreyLevelEmphasis_angle45_offset1"

"HighGreyLevelRunEmphasis_AllDirection_offset4_SD"

"Elongation"

LASSO, least absolute shrinkage selection operator.

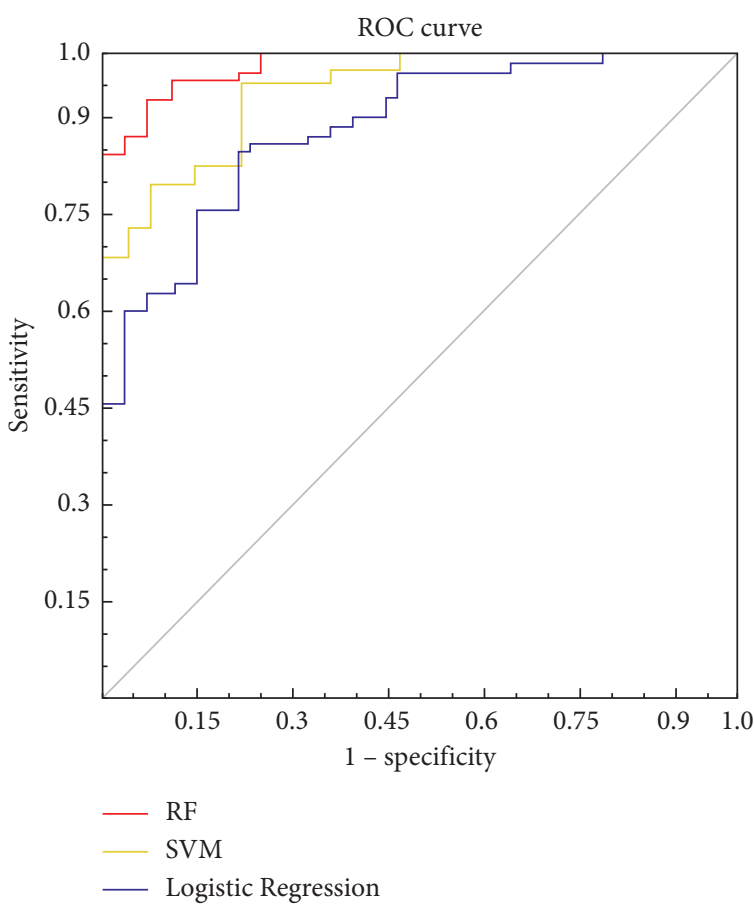

(a)

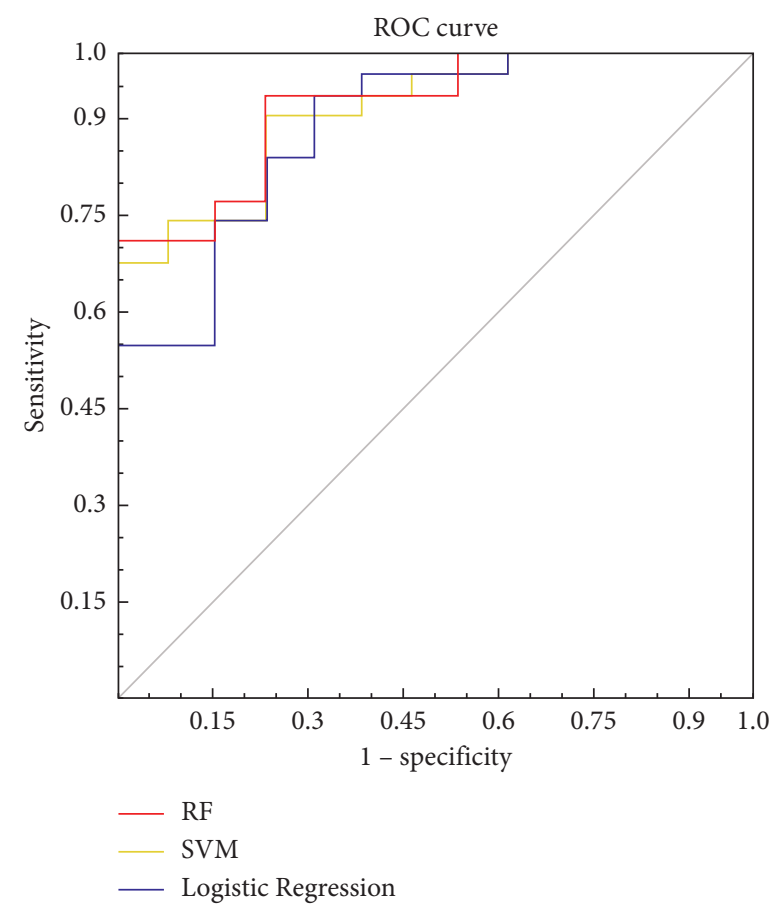

(b)

FIGURE 5: Continued. 


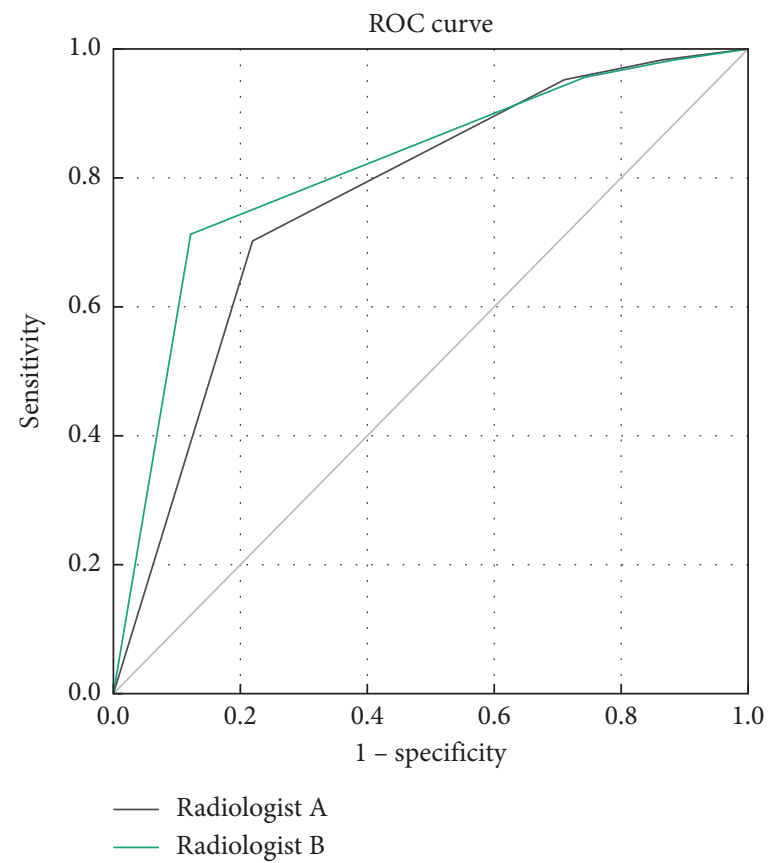

(c)

FIGURE 5: ROC curves of the radiomics signature in the training (a) and test (b) cohorts. ROC curves for the radiomics-based ADC and T2WI model and PI-RADS (c) score performance in distinguishing low- vs. high-grade PCa in the training and test groups. RF, random forest tree; SVM, support vector machine. Predictive ability of the PI-RADS V 2.1 model.

TABLE 4: Discrimination for differentiating low-grade and high-grade PCa in the training and test group and PI-RADS V2.1 scores.

\begin{tabular}{|c|c|c|c|c|c|c|c|}
\hline Model & Machine learning & AUC & Accuracy & Sensitivity & Specificity & $\mathrm{LR}+$ & LR- \\
\hline \multirow{3}{*}{ T2WI combine ADC (training) } & $\mathrm{RF}$ & 0.982 & 0.857 & 0.92 & 0.714 & 3.2168 & 0.112 \\
\hline & Logistic regression & 0.886 & 0.847 & 0.968 & 0.538 & 2.0952 & 0.0595 \\
\hline & SVM & 0.943 & 0.857 & 0.957 & 0.621 & 2.5251 & 0.0692 \\
\hline \multirow{3}{*}{ T2WI combine ADC (test) } & $\mathrm{RF}$ & 0.918 & 0.795 & 0.935 & 0.462 & 1.7379 & 0.141 \\
\hline & Logistic regression & 0.886 & 0.841 & 0.968 & 0.538 & 2.0952 & 0.06 \\
\hline & SVM & 0.913 & 0.841 & 0.935 & 0.615 & 2.4286 & 0.106 \\
\hline \multirow{2}{*}{ PI-RADS V2.1 } & Reader 1 & 0.767 & 0.725 & 0.703 & 0.78 & 3.1955 & 0.381 \\
\hline & Reader 2 & 0.813 & 0.76 & 0.713 & 0.878 & 5.8443 & 0.327 \\
\hline
\end{tabular}

ADC, apparent diffusion coefficient; T2WI, T2-weighting imaging; AUC, area under the curve; RF, random forest tree; SVM, support vector machine; LR+, positive likelihood ratio; LR-, negative likelihood ratio; PI-RADS V2.1, prostate imaging reporting and data system version 2.1.

Table 5: Performance of each of the radiologists in the PI-RADS V2.1 scoring of the high-grade PCa group and the low-grade PCa group.

\begin{tabular}{lcccc}
\hline \multirow{2}{*}{ PI-RADS V2.1scores } & \multicolumn{2}{c}{ High-grade PCa } & \multicolumn{2}{c}{ Low-grade PCa } \\
& Radiologist A & Radiologist B & Radiologist A & 3 \\
Radiologist B \\
\hline 1 & 1 & 0 & 3 & 0 \\
2 & 1 & 2 & 6 & 5 \\
3 & 3 & 3 & 20 & 26 \\
4 & 25 & 25 & 9 & 5 \\
5 & 71 & 72 & 41 & 41 \\
Total & 101 & 101 & & 5 \\
\hline
\end{tabular}

PI-RADS V2.1, prostate imaging reporting and data system version 2.1; PCa, prostate cancer.

established an mp-MRI-based radiomics model for discriminating between clinically significant PCa and clinically insignificant PCa with an AUC of 0.823 [34]. However, these research methods only extract a few texture features or only use one machine learning model and are not compared with the traditional PI-RADS V2.1 score, and the feedback information is relatively limited. In the current study, three classifiers were utilized and compared with the PI-RADS V2.1 score; then, the most effective features were selected for the construction of the three prediction models. 
bp-MRI allows for accurate detection and localization of suspicious $\mathrm{PCa}$, in addition to a reduction of the time required to complete the study and lower costs without using gadolinium [38]. Moreover, potential risks related to the use of gadolinium-based contrast media, such as nephrogenic systemic fibrosis, renal failure, and accumulation of gadolinium in the brain, would be reduced [39]. In general, T2WI and ADC are the most common sequences selected by investigators for radiomics research [32, 40-42]. Therefore, we chose to derive the radiomic features from T2WI and ADC only for this study. In addition, using too many sequences affects clinical application because of timeconsuming and laborious image segmentation, so it is more important to choose a valuable sequence. Therefore, we conclude that mp-MRI-based radiomics may contribute to differentiating low-grade from high-grade PCa and to risk stratification without the need for additional MRI sequences such as DCE.

High-grade PCa with more heterogeneous tissue conditions than low-grade PCa tumors exhibited on the spatial distribution of voxel intensities and radiomics could provide more information to distinguish cancers that are challenging for the PI-RADS V2.1 score [42, 43]. In addition, the clinical application of PI-RADS V2.1 is subjective and highly readerdependent due to its natural feature of strictly fixed discrete categories. Moreover, PI-RADS V2.1 requires a DCE sequence and has the disadvantage of achieving quantitative criteria that favor diagnostic performance. In this study, we proposed automatic machine learning approaches to address these challenges and employed a well-recognized method, $\mathrm{RF}$, leading to robust classifier performance without using gadolinium. RF is an ensemble algorithm with high accuracy and tolerance [44]. Its greatest advantage is that it can predict multivariate data and analyze complex nonlinear relationships [23].

However, there were also some limitations in this study. First, it was a retrospective single-center study, and the sample size was relatively small. Prospective multiple-center studies are needed in the future, and other external validation cohorts should also be included to test the reproducibility of future studies. Second, although a three-dimensional VOI was used, the deviation caused by subjective factors is inevitable when the VOI is manually drawn. We manually segmented and delineated the VOIs with the two radiologists in consensus, trying our best to reduce the deviation. Further studies should adopt semiautomatic segmentation. Third, some pathological results were proven by systematic combined targeted biopsy and lacked whole-mount serial section. Although wholemount histopathology is considered the reference standard, it is unreasonable to expect that all of our cases would undergo radical prostatectomy, especially for low-grade PCa patients. Additionally, only including patients who underwent radical prostatectomy would lead to selection bias.

\section{Conclusion}

In summary, our study shows that machine learning-based analysis of the bp-MRI radiomic model presented superior diagnostic performance to traditional PI-RADS V2.1 scores for predicting the histological grade of PCa. The machine learning algorithm RF model was slightly increased. It was found that the algorithm assists radiologists in reporting tumor grade and facilitates clinical decision-making for the management of PCa.

\section{Data Availability}

The data used to support the findings of this study are included within the Supplementary Information file.

\section{Conflicts of Interest}

The authors declare that there are no conflicts of interest regarding the publication of this article.

\section{Supplementary Materials}

The supplementary file contains the supplementary information about the original data for radiomics (Supplementary Materials and Supplement Tables 1-6). (Supplementary Materials)

\section{References}

[1] R. L. Siegel, K. D. Miller, H. E. Fuchs, and A. Jemal, "Cancer statistics, 2021," CA: A Cancer Journal for Clinicians, vol. 71, no. 1, pp. 7-33, 2021.

[2] P. C. Walsh, T. L. DeWeese, and M. A. Eisenberger, "Localized prostate cancer," New England Journal of Medicine, vol. 357, no. 26, pp. 2696-2705, 2007.

[3] G. Draisma, R. Boer, I. W. van der Cruijsen, R. A. M. Damhuis, F. H. Schroder, and H. J. de Koning, "Lead times and overdetection due to prostate-specific antigen screening: estimates from the European Randomized Study of Screening for Prostate Cancer," JNCI Journal of the National Cancer Institute, vol. 95, no. 12, pp. 868-878, 2003.

[4] R. C. Macefield, C. Metcalfe, C. Metcalfe et al., "Impact of prostate cancer testing: an evaluation of the emotional consequences of a negative biopsy result," British Journal of Cancer, vol. 102, no. 9, pp. 1335-1340, 2010.

[5] L. Klotz, L. Zhang, A. Lam, R. Nam, A. Mamedov, and A. Loblaw, "Clinical results of long-term follow-up of a large, active surveillance cohort with localized prostate cancer," Journal of Clinical Oncology, vol. 28, no. 1, pp. 126-131, 2010.

[6] "NICE Prostate cancer: diagnosis and treatment," 2015, http:// www.nice.org.uk/guidance/CG175.

[7] N. Mottet, R. C. N. van den Bergh, E. Briers, and Expert Patient Advocate (European Prostate Cancer Coalition/Europa UOMO), "European association of urology: prostate cancer guidelines," 2019, https://uroweb.org/wp-content/ uploads/Prostate-Cancer-2018-pocket.pdf.

[8] H. U. Ahmed, A. El-Shater Bosaily, R. Gabe et al., "Diagnostic accuracy of multi-parametric MRI and TRUS biopsy in prostate cancer (PROMIS): a paired validating confirmatory study," The Lancet, vol. 389, no. 10071, pp. 815-822, 2017.

[9] B. Turkbey, A. B. Rosenkrantz, M. A. Haider et al., "Prostate imaging reporting and data system version 2.1: 2019 update of prostate imaging reporting and data system version 2," $E$ ropean Urology, vol. 76, no. 3, pp. 340-351, 2019.

[10] R. Cuocolo, M. B. Cipullo, A. Stanzione et al., "Machine learning for the identification of clinically significant prostate 
cancer on MRI: a meta-analysis," European Radiology, vol. 30, no. 12, pp. 6877-6887, 2020.

[11] T. J. M. Castillo, M. P. A. Starmans, M. Arif et al., "A multicenter, multi-vendor study to evaluate the generalizability of a radiomics model for classifying prostate cancer: high grade vs. Low grade," Diagnostics, vol. 11, no. 2, p. 369, 2021.

[12] S. Transin, R. Souchon, C. Gonindard-Melodelima et al., "Computer-aided diagnosis system for characterizing ISUP grade $\geq 2$ prostate cancers at multiparametric MRI: a crossvendor evaluation," Diagnostic and interventional imaging, vol. 100, no. 12, pp. 801-811, 2019.

[13] M. Scialpi, E. Prosperi, A. D’andrea et al., "Biparametric versus multiparametric MRI with non-endorectal coil at 3T in the detection and localization of prostate cancer," Anticancer Research, vol. 37, no. 3, pp. 1263-1271, 2017.

[14] M. Scialpi, V. Rondoni, M. C. Aisa et al., "Is contrast enhancement needed for diagnostic prostate MRI?" Translational Andrology and Urology, vol. 6, no. 3, pp. 499-509, 2017.

[15] X. Wu, P. Reinikainen, M. Kapanen, T. Vierikko, P. Ryymin, and P.-L. Kellokumpu-Lehtinen, "Dynamic contrast-enhanced imaging as a prognostic tool in early diagnosis of prostate cancer: correlation with PSA and clinical stage," Contrast Media and Molecular Imaging, vol. 19, Article ID 3181258, 2018.

[16] H. Meyer, W. Andreas, and S. Alexey, "Can dynamic contrast enhanced MRI predict gleason score in prostate cancer? a systematic review and meta analysis," Urologic Oncology: Seminars and Original Investigations, vol. 39, 2021.

[17] T. Chen, M. Li, Y. Gu et al., "Prostate cancer differentiation and aggressiveness: assessment with a radiomic-based model vs. PI-RADS v2," Journal of Magnetic Resonance Imaging, vol. 49, no. 3, pp. 875-884, 2019.

[18] R. J. Gillies, P. E. Kinahan, and H. Hricak, "Radiomics: images are more than pictures, they are data," Radiology, vol. 278, no. 2, pp. 563-577, 2016.

[19] B. Abraham and M. S. Nair, "Computer-aided diagnosis of clinically significant prostate cancer from MRI images using sparse autoencoder and random forest classifier," Biocybernetics and Biomedical Engineering, vol. 38, no. 3, pp. 733-744, 2018.

[20] N. Dikaios, J. Alkalbani, M. Abd-Alazeez et al., "Zone-specific logistic regression models improve classification of prostate cancer on multi-parametric MRI," European Radiology, vol. 25, no. 9, pp. 2727-2737, 2015.

[21] M. M. López, J. Ramírez, J. M. Górriz et al., "SVM-based CAD system for early detection of the Alzheimer's disease using kernel PCA and LDA," Neuroscience Letters, vol. 464, no. 3, pp. 233-238, 2009.

[22] B. Turkbey and P. L. Choyke, "Future perspectives and challenges of prostate MR imaging," Radiologic Clinics of North America, vol. 56, no. 2, pp. 327-337, 2018.

[23] A. G. Chung, F. Khalvati, M. J. Shafiee, M. A. Haider, and A. Wong, "Prostate cancer detection via a quantitative radiomics-driven conditional random field framework," IEEE Access, vol. 3, pp. 2531-2541, 2015.

[24] A. Cameron, F. Khalvati, M. A. Haider, and A. Wong, "MAPS: a quantitative radiomics approach for prostate cancer detection," IEEE Transactions on Biomedical Engineering, vol. 63, no. 6, pp. 1145-1156, 2015.

[25] L. Liu, Z. Tian, Z. Zhang, and B. Fei, "Computer-aided detection of prostate cancer with MRI: technology and applications," Academic Radiology, vol. 23, no. 8, pp. 1024-1046, 2016.

[26] B. Feng, M. Zhang, H. Zhu, L. Wang, and Y. Zheng, "MRI image segmentation model with support vector machine algorithm in diagnosis of solitary pulmonary nodule," Contrast Media \& Molecular Imaging, vol. 2021, Article ID 9668836, 2021.

[27] Y. Zhang, W. Zhao, C. Wei et al., "The value of biparametric MRI in the detection of prostate cancer," Chinese Journal of Radiology, vol. 12, pp. 109-114, 2019.

[28] J. Cohen, "A coefficient of agreement for nominal scales," Educational and Psychological Measurement, vol. 20, no. 1, pp. 37-46, 1960.

[29] E. R. DeLong, D. M. DeLong, and D. L. Clarke-Pearson, "Comparing the areas under two or more correlated receiver opening characteristic curves: an on parametric approach," Biometrics, vol. 44, no. 3, pp. 837-845, 1988.

[30] P. De Visschere, N. Lumen, P. Ost, K. Decaestecker, E. Pattyn, and G. Villeirs, "Dynamic contrast-enhanced imaging has limited added value over T2-weighted imaging and diffusionweighted imaging when using PI-RADSv2 for diagnosis of clinically significant prostate cancer in patients with elevated PSA," Clinical Radiology, vol. 72, no. 1, pp. 23-32, 2017.

[31] D. Kwon, I. M. Reis, A. L. Breto et al., "Classification of suspicious lesions on prostate multiparametric MRI using machine learning," Journal of Medical Imaging, vol. 5, no. 3, Article ID 034502, 2018.

[32] J. T. Kwak, S. Xu, B. J. Wood et al., "Automated prostate cancer detection using T2-weighted and high-b-value diffusion-weighted magnetic resonance imaging," Medical Physics, vol. 42, no. 5, pp. 2368-2378, 2015.

[33] S. K. Majumder, N. Ghosh, and P. K. Gupta, "Support vector machine for optical diagnosis of cancer," Journal of Biomedical Optics, vol. 10, no. 2, p. 024034, 2005.

[34] X. Min, M. Li, D. Dong et al., "Multi-parametric MRI-based radiomics signature for discriminating between clinically significant and insignificant prostate cancer: cross-validation of a machine learning method," European Journal of Radiology, vol. 115, pp. 16-21, 2019.

[35] N. A. Parra, H. Lu, Q. Li et al., "Predicting clinically significant prostate cancer using DCE-MRI habitat descriptors," Oncotarget, vol. 9, no. 98, pp. 37125-37136, 2018.

[36] S. Ma, H. Xie, H. Wang et al., "MRI-based radiomics signature for the preoperative prediction of extracapsular extension of prostate cance," Journal of Magnetic Resonance Imaging, vol. 50, no. 6, pp. 1914-1925, 2019.

[37] X.-k. Niu, Z.-f. Chen, L. Chen, J. Li, T. Peng, and X. Li, "Clinical application of biparametric MRI texture analysis for detection and evaluation of high-grade prostate cancer in zone-specific regions," American Journal of Roentgenology, vol. 210, no. 3, pp. 549-556, 2018.

[38] M. Scialpi, A. D’Andrea, E. Martorana et al., "Biparametric MRI of the prostate," Türk Üroloji Dergisi/Turkish Journal of Urology, vol. 43, no. 4, pp. 401-409, 2017.

[39] R. J. McDonald, J. S. McDonald, D. F. Kallmes et al., "Intracranial gadolinium deposition after contrast-enhanced MR imaging," Radiology, vol. 275, no. 3, pp. 772-782, 2015.

[40] F. Khalvati, A. Wong, and M. A. Haider, "Automated prostate cancer detection via comprehensive multi-parametric magnetic resonance imaging texture feature models," $B M C$ Medical Imaging, vol. 15, no. 1, pp. 27-14, 2015.

[41] A. Stanzione, R. Cuocolo, S. Cocozza et al., "Detection of extraprostatic extension of cancer on biparametric MRI combining texture analysis and machine learning: preliminary results," Academic Radiology, vol. 26, no. 10, pp. 1338-1344, 2019.

[42] J. V. Hegde, R. V. Mulkern, L. P. Panych et al., "Multiparametric MRI of prostate cancer: an update on state-of-the-art techniques 
and their performance in detecting and localizing prostate cancer," Journal of Magnetic Resonance Imaging, vol. 37, no. 5, pp. 1035-1054, 2013.

[43] O. F. Donati, Y. Mazaheri, A. Afaq et al., "Prostate cancer aggressiveness: assessment with whole-lesion histogram analysis of the apparent diffusion coefficient," Radiology, vol. 271, no. 1, pp. 143-152, 2014.

[44] L. Breiman, "Random forests," Machine Learning, vol. 45, no. 1, pp. 5-32, 2001. 